\title{
Study on Environmental Impact Calculation of 500kV UHV AC \\ Transmission Line Power Frequency Electric Field
}

\author{
Zhining Wang ${ }^{1, a}$, Zhang Chen ${ }^{2, b}$ \\ ${ }^{1}$ Wuhan Institute of Marine Electric Propulsion, CSIC, Wuhan, China \\ ${ }^{2}$ Hubei Provincial Center for Quality Supervision and Test of Construction \\ Engineering,Wuhan,China \\ a1321662628@qq.com, b939306080@qq.com
}

Keywords: Environment effect, Electric field strength, High voltage transmission line, Theoretical calculation

\begin{abstract}
The theoretical calculation formula of the power frequency electric field around the high voltage AC transmission line is improved through the research, and comparing the calculated results with the measured results of the 500kV UHV AC transmission line which has been built, the improved theory between calculated and measured values was the same trend, the same precision, and the theoretical value is slightly larger than the measured. It is practical and valuable to predict and analyze the influence of power frequency electric field on the surrounding environment of high voltage AC transmission line.
\end{abstract}

\section{Introduction}

Along with the expansion of the scale and voltage level of high voltage AC transmission line, the influence of transmission line on the people and the environment is also concerned[1].The traditional two dimensional electric field intensity has a good application in the calculation of the electric field intensity of the symmetrical line. However, with the increase of the accuracy of the calculation and some special transmission lines, the theoretical calculation results of the traditional two-dimensional electric field strength calculation formula are greatly different from the actual measurement results, and it is not applicable anymore[2].In order to satisfy the high voltage AC transmission lines electric field strength calculation accuracy,consistency and difficulty, it is necessary to optimize the traditional formula to ensure the reliability of the environmental impact prediction at the design stage.

\section{Improvement of calculation formula}

\section{Calculate line equivalent charge}

Assuming that the coordinates of any equivalent charge $\mathrm{Q}$ on a conductor in space are $\left(\mathrm{x}_{1}, \mathrm{y}_{1}, \mathrm{z}_{1}\right)$, according to the Maxwell formula, the relationship between the ground voltage and the equivalent charge on the high voltage $\mathrm{AC}$ transmission line is:

$$
\mathrm{Q}=\mathrm{P}^{-1} \mathrm{U}
$$

In which $\mathrm{Q}$ is the matrix of equivalent charge on the transmission line; $\mathrm{U}$ is the equivalent charge on the transmission line on the ground voltage matrix; $\mathrm{P}$ is the transmission line of the equivalent charge of the potential coefficient matrix [3,4].

Without considering the effect of the earth on the equivalent charge of the transmission line, the 
potential coefficient $\mathrm{P}$ of any equivalent charge in space can be approximated as:

$$
P=\frac{1}{4 \pi \varepsilon R}
$$

For $\pi$ and $\varepsilon$ type is constant, $\mathrm{R}$ is an arbitrary space $\mathrm{A}(\mathrm{x}, \mathrm{y}, \mathrm{z})$ to the equivalent of a single circular cylinder conductor wire in the distance, $\mathrm{R}$ approximate formula can be expressed as:

$$
R=\sqrt{\left(x-x_{1}\right)^{2}+\left(y-y_{1}\right)^{2}+\left(z-z_{1}\right)^{2}-\left(d-d_{e q}\right)^{2}}
$$

In which $\mathrm{d}$ is the geometric radius of the split conductor, Unit: $\mathrm{M}$; $d_{e q}$ is the equivalent of a single circular cylinder conductor radius, Unit: M. And $d_{e q}$ can be calculated according to the following formula:

$$
d_{e q}=d\left(\frac{n r}{d}\right)^{\frac{1}{n}}
$$

In which $\mathrm{n}$ is the number of bundled conductors, $\mathrm{r}$ is the split conductor radius, Unit: $\mathrm{M}$.

\section{Calculation of electric field strength}

After calculating the equivalent charge on the transmission line, we can calculate the field strength of any point in the space according to the superposition principle. Assuming that the Three-dimensional coordinate system is established, The $\mathrm{X}$ axis is parallel to the transmission line, The $\mathrm{Y}$ axis is perpendicular to the transmission line, The $\mathrm{Z}$ axis is perpendicular to the transmission line and the ground surface, then the electric field strength component at point $A(x, y, z)$ is:

$$
\begin{aligned}
& E_{\mathrm{x}}=\frac{1}{4 \pi \varepsilon} \sum_{i=1}^{m} Q_{i}\left(\frac{x-x_{i}}{R^{2}}-\frac{x-x_{i}}{R^{\prime 2}}\right) \\
& E_{\mathrm{y}}=\frac{1}{4 \pi \varepsilon} \sum_{i=1}^{m} Q_{i}\left(\frac{y-y_{i}}{R^{2}}-\frac{y-y_{i}}{R^{2}}\right) \\
& E_{z}=\frac{1}{4 \pi \varepsilon} \sum_{i=1}^{m} Q_{i}\left(\frac{z-z_{i}}{R^{2}}-\frac{z-z_{i}}{R^{2}}\right)
\end{aligned}
$$

In which $\mathrm{m}$ is the number of transmission line, ${ }_{i} 、 y_{i}, z_{i}$ as the equivalent charge coordinate traverse $\mathrm{i}, R^{\prime}$ is the distance between a point $\mathrm{A}$ and Mirror equivalent charge of the transmission line.

The size of the field strength at the A point that can be calculated by the above formula is:

$$
E=\sqrt{E_{\mathrm{x}}^{2}+E_{y}^{2}+E_{z}^{2}}
$$

\section{Calculation accuracy comparison}

In order to simplify the comparison with the traditional three dimensional theory,the electric field generated by an infinite charge is studied here. The selection of the Three dimensional coordinate system is: parallel to the ground surface but perpendicular to the direction of the transmission line is the $\mathrm{X}$ axis direction, perpendicular to the ground and the transmission line direction as $\mathrm{Y}$ axis direction, parallel to the ground and the transmission line direction as $\mathrm{Z}$ axis direction. According to 
the above assumptions and the coordinates of selected point $\mathrm{A}$, equivalent charge coordinates and $\mathrm{d} 、 \mathrm{n}$, it can be known that $E_{\mathrm{z}}=0$. Compared with the theoretical calculation of the traditional three dimensional theory, the results are shown in Table 1.

Table 1 Theoretical results contrast

\begin{tabular}{ccc}
\hline & Improved Three Dimensional & Traditional Three Dimensional \\
& Theoretical Solution $\left(E_{\mathrm{x}}, E_{\mathrm{y}}\right)$ & Theoretical Solution $\left(E_{\mathrm{x}}, E_{y}\right)$ \\
\hline$(2.25,3.32,2.59)$ & $(2.179123,3.186324)$ & $(2.157831,3.160254)$ \\
$(2.43,4.23,1.95)$ & $(1.498123,2.698754)$ & $(1.503621,2.709875)$ \\
$(3.00,3.28,1.42)$ & $(2.584321,2.520031)$ & $(2.567852,2.523684)$ \\
$(3.20,2.55,1.02)$ & $(3.035687,2.401562)$ & $(3.027865,2.396521)$ \\
$(3.66,2.58,1.46)$ & $(2.906895,2.065213)$ & $(2.913965,2.076542)$ \\
$(4.43,1.83,1.00)$ & $(2.763564,1.356214)$ & $(2.759874,1.336985)$ \\
$(5.51,3.75,2.23)$ & $(2.036548,1.312652)$ & $(2.035132,1.298547)$ \\
$(6.45,6.42,3.62)$ & $(1.204523,1.032014)$ & $(1.225675,1.023527)$ \\
$(7.08,8.20,2.05)$ & $(0.953214,0.987541)$ & $(0.912356,0.975641)$ \\
\hline
\end{tabular}

Compared with the traditional three-dimensional calculation results, the results calculated by the improved three-dimensional theoretical formula are the same as the traditional three dimensional calculation accuracy,and the $E_{\mathrm{x}}$ and $E_{y}$ calculated by the improved three-dimensional electric field strength calculation formula are generally higher than the traditional three-dimensional electric field strength theory calculated results,it is very favorable and practical for the forecast of the design phase of UHV AC transmission line environmental impact.

\section{Example verification}

In order to verify the relationship between the theoretical calculation results and the actual measurement results, this paper selects a 500kV UHV AC transmission line which has been built to conduct a comparative analysis of measured. The distance from the single return conductor in the $500 \mathrm{kV}$ UHV AC transmission lines to ground is $16.5 \mathrm{~m}$, the wires are arranged horizontally and the line spacing is $12 \mathrm{~m}$, the distance from the double circuit conductor to ground is $16.2 \mathrm{~m}$. The wire level spacing is $13 \mathrm{~m}$ (Upper phase conductor), $17 \mathrm{~m}$ (Medium phase conductor), $15 \mathrm{~m}$ (bottom phase conductor), the vertical line spacing was $10.6 \mathrm{~m} / 10.7 \mathrm{~m}$. According to the field measurement data and theoretical calculation formula, the results of the $500 \mathrm{kV}$ UHV AC transmission lines are summarized in Table 2. 
Table 2 The 500kV UHV AC transmission lines

electric field intensity measured results and calculated results comparison table

\begin{tabular}{|c|c|c|}
\hline \multirow{2}{*}{$\begin{array}{c}\text { Distance from the center of } \\
\text { the line }(m)\end{array}$} & \multicolumn{2}{|c|}{$\mathbf{E}(\mathbf{k V} / \mathbf{m})$} \\
\hline & Calculated results & Measured results \\
\hline 0 (Medium phase conductor) & 2.48 & 3.05 \\
\hline 1 & 2.53 & 3.15 \\
\hline 2 & 2.70 & 3.20 \\
\hline 3 & 2.95 & 3.20 \\
\hline 4 & 3.15 & 3.56 \\
\hline 5 & 3.48 & 3.45 \\
\hline 6 & 3.73 & 3.34 \\
\hline 7 & 3.91 & 3.55 \\
\hline 8 & 4.12 & 3.65 \\
\hline 9 & 4.25 & 3.89 \\
\hline 10 (Edge phase conductor) & 4.31 & 4.25 \\
\hline 11 & 4.27 & 4.10 \\
\hline 12 & 4.15 & 4.01 \\
\hline 13 & 4.06 & 3.95 \\
\hline 14 & 3.90 & 3.88 \\
\hline 15 & 3.74 & 3.61 \\
\hline 16 & 3.57 & 3.37 \\
\hline 17 & 3.35 & 3.10 \\
\hline 18 & 3.14 & 2.91 \\
\hline 19 & 2.88 & 2.91 \\
\hline 20 & 2.64 & 2.63 \\
\hline 21 & 2.45 & 2.41 \\
\hline 22 & 2.27 & 2.04 \\
\hline 23 & 2.01 & 1.75 \\
\hline 24 & 1.86 & 1.47 \\
\hline 25 & 1.71 & 1.32 \\
\hline 26 & 1.55 & 1.28 \\
\hline 27 & 1.39 & 1.17 \\
\hline 28 & 1.26 & 1.18 \\
\hline 29 & 1.18 & 1.02 \\
\hline 30 & 1.05 & 0.99 \\
\hline 35 & 0.67 & 0.65 \\
\hline 40 & 0.38 & 0.41 \\
\hline 50 & 0.15 & 0.16 \\
\hline 60 & 0.06 & 0.05 \\
\hline
\end{tabular}

The comparison between the calculated results and measured results of $500 \mathrm{kV}$ UHV AC transmission line power frequency electric field strength as shown in Figure 1: 


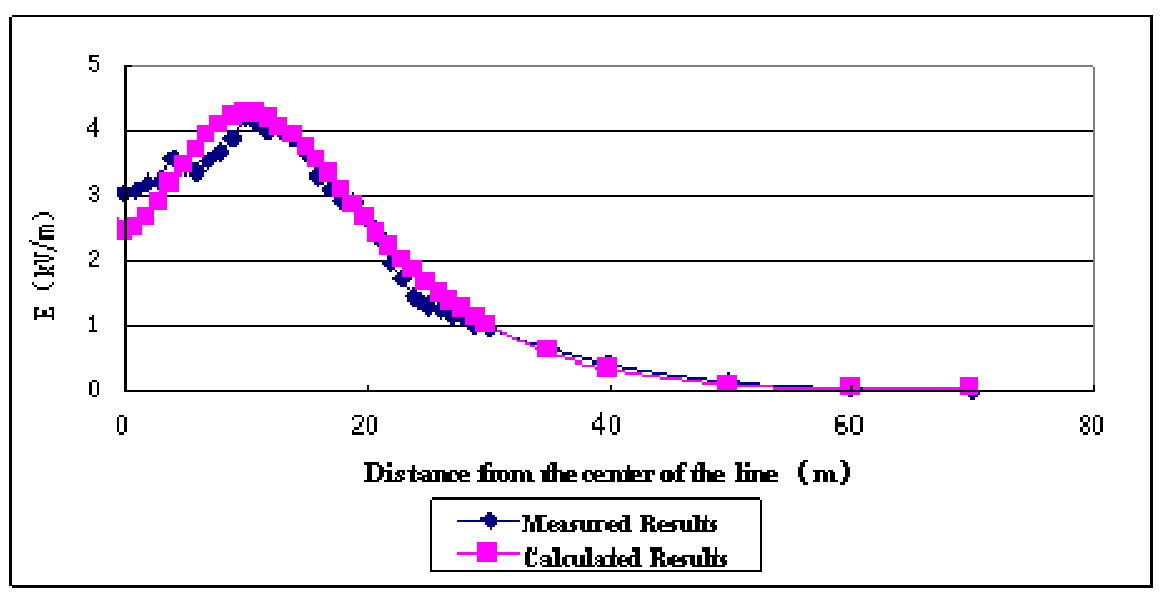

Fig 1 Comparison between the measured results and calculated results

As shown in Table 2 and Figure 1,the calculated results are in agreement with the measured results, the power frequency electric field intensity variation trend is consistent. The theoretical calculation of power frequency electric field maximum value is $4.31 \mathrm{kV} / \mathrm{m}$, and it appears in the edge phase conductor projection. The measured results of power frequency electric field maximum value is $4.25 \mathrm{kV} / \mathrm{m}$, and also appears in the edge phase conductor projection. The calculated maximum result is slightly larger than the actual measured result.

\section{Conclusions}

(1) Based on the theoretical calculation formula of the electric field strength of the traditional high voltage AC transmission lines for the improvement, the traditional two-dimensional calculation formula is improved to the three-dimensional computing formula, the calculation method is simple and easy to use, and it has the engineering application value in improving the measurement precision.

(2) In order to further verify the theoretical formula of the electric field strength after the improvement of the accuracy and its practical application value, the 500kV UHV AC transmission line which has been built is selected for measurement, the theoretical calculation results can satisfy the requirements of the actual measurement accuracy and the trend of change by comparing and analyzing the theoretical calculation results and the actual measurement results. From the analysis of the data, it can be seen that the theoretical calculation results are generally larger than the measured results, and it has a good practical value for predicting the influence of the power frequency electric field of the high voltage AC transmission line on the surrounding environment in the design stage.

\section{References}

[1] Wu Jingru,Xu Yongxi. Development prospect of UHV AC power transmission in China[J].Power System Technology,2005,29(3):1-4.(In Chinese)

[2] Ma Na,Zou Peng, Zhang Haidong, Zhang Yage. Mathematical model and simulation of power frequency electric field on high voltage transmission lines programmed by MATLAB[J]Micro Computer Information,2009,07:210-211+198. (In Chinese)

[3] Shao Fangyin.Conductor configuration and related characteristics of $500 \mathrm{kV}$ compact transmission lines[J].Electric Power,1996,29(2):3-7. (In Chinese)

[4] Zhou Haoyu,Yu Hong.Discussion on several important problems of developing UHV AC transmission in China[J]. Power System Technology,2005,29(12):1-9. (In Chinese) 